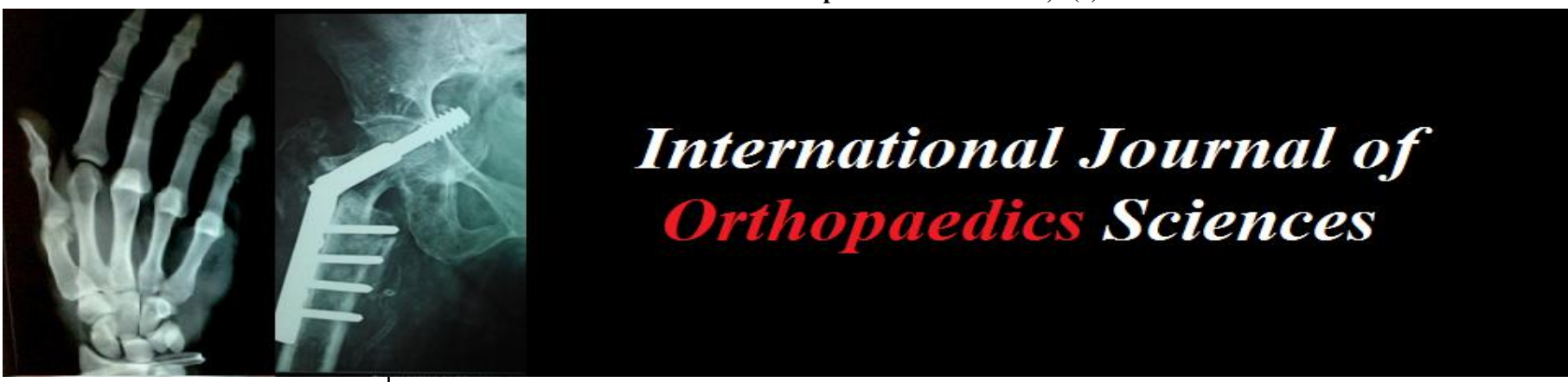

ISSN: $2395-1958$

IJOS 2018; 4(1): 1025-1030

(C) 2018 IJOS

www.orthopaper.com

Received: 20-11-2017

Accepted: 23-12-2017

Dr. Arpit Jariwala

FRCS, Ortho, U.K

Dr. Mir Zia Ur Rahman Ali MCH Ortho (U.K), Consultant Pediatric Surgeon, Thumby Hospital, Chaderghat,

Hyderabad, Telangana, India
Correspondence

Dr. Mir Zia Ur Rahman Ali MCH Ortho (U.K),

Consultant Pediatric Surgeon, Thumby Hospital, Chaderghat, Hyderabad, Telangana, India

\section{Patients perception of outcome in arthroscopic subacromial decompression using proms scores}

\author{
Arpit Jariwala and Mir Zia Ur Rahman Ali
}

DOI: https://doi.org/10.22271/ortho.2018.v4.110.146

\section{Abstract}

Subacromial impingement syndrome (SIS) is the most common cause of shoulder pain, with higher incidence in middle age and elderly population; this will have an impact upon their activities of daily living and quality of life. Aim was to assess the outcome of Arthroscopic subacromial decompression (ASAD) based on patient's perception of their outcome using patient reported outcome measure scores (PROMs). 50 patients with SIS, who underwent ASAD from April 2015 to January 2017 at NHS Tayside, who were part of Tayside Upper Limb Evaluation Programme (TULEP) were included in this study. Improvement in mean postoperative PROMs scores at 6 months and 1 year postoperative was observed, with maximum improvement at 6 month postoperative period, however, less improvement was noted from 6 months to 1 year postoperatively which was statistically significant. Improvement in PROMs postoperatively was better appreciated inpatients aged less than 50 years, females, low BMI, and left shoulder pathology. Patients who were dissatisfied at 6 months postoperatively had continued to score less at 1 year postoperative. Pain was the most common reason for dissatisfaction. This study suggested that ASAD for impingement gives a high patient satisfaction with significant improvement in function and quality of life.

Keywords: Subacromial impingement syndrome (SIS), shoulder pain, arthroscopic subacromial decompression (ASAD), patient reported outcome measures (PROMs), EQ 5D 3L, Quick DASH, Oxford shoulder score

\section{Introduction}

Subacromial impingement syndrome (SIS) is the most common cause of shoulder pain seen by upper limb surgeons. It includes spectrum of pathology and is caused by entrapment of rotator cuff and bursa between the anteroinferior edge of acromion and coracoacromial arch when the arm is elevated. ${ }^{[1]}$ It is a major cause of morbidity and disability in the general population with the prevalence of shoulder pain in United Kingdom (UK) population currently being estimated to be between $16 \%$ to $26 \%$, shoulder problems are responsible for $2.4 \%$ of all United Kingdom's General Physician (GP) consultation. ${ }^{[2]}$ The incidence is higher in middle age and elderly population, this will have an impact upon their activities of daily living and quality of life. Management for the patients with subacromial impingement ranges from conservative, open surgical to arthroscopic management. Arthroscopic subacromial decompression (ASAD) has become a widely used technique for the treatment of SIS and is increasingly in UK with epidemiological data showing that intervention rates were increasing from 5.2 per 100,000 in 2001-2002 to 40.2 per 100,000 in $2009-2010$. ${ }^{[3]}$ In recent years, a change towards a whole subjective person's perception in evaluation of a treatment has been taken into practice.

In view of the increasing number of surgical interventions and the controversies, it was of vital importance to understand whether ASAD can improve quality of life of the patient. ${ }^{[4]}$ The quality adjusted life year (QALY) was an internationally accepted measure of health gain taking into account quality and quantity of health. QALY is currently practised by NICE in cost building analysis of health interventions. It can be evaluated by various tools including EQ5 D 3L, OSS and QD being the preferred method for health and care excellence in UK. ${ }^{[4]}$ The present study is different from the previous studies because it had considered three subjective scoring systems to analyse the outcome of health gain and quality of life after ASAD. We analysed all the parameters in the scoring system to assess the health gain. 
This eventually gave a detail assessment of overall health of the individual after surgery. In this study, we have assessed the outcome of the ASAD considering PROMs as a marker of improvement in quality of life. We considered the EQ5D, OSS and Quick DASH scores to reflect the outcome of surgery. These scores had been validated and accepted for evaluating the health gain considering quality of health. ${ }^{[4]}$ More recently focus has shifted towards PROMs to ascertain the patient's perception of success. A PROM was any measure reported by the patient in a validated standardised format. Proponents of these suggested that they provide a remarkably sophisticated measure of whether a patient feels better and how much better. ${ }^{[5]}$ Allom et al. ${ }^{[6]}$ stated that DASH and OSS scores were effective scoring tools, providing good indicators of post-operative outcome for disorders of the rotator cuff. They demonstrated that both the DASH and the Constant scores as well as the Oxford and Constant scores were similar in assessing post-operative outcome. Hultenheim Klintberg et al. ${ }^{[7]}$ looked into patient satisfaction and physical activity at 8 to 11 years after ASAD. They reported positive results in their long-term study with a high level of healthrelated quality of life, patient satisfaction and physical activity 8-11 years after ASAD.

\section{Methods and Materials}

A total of 50 patients who underwent ASAD at NHS Tayside Hospital Trust were studied. The study was a prospective analysis from Tayside Upper Limb Evaluation Programme (TULEP) database for analysis of surgical outcome of ASAD among patients who underwent surgery at NHS Tayside Hospitals form April of 2015 to January 2017 patients with SIS. Pre-operative data was collected using questionnaires, after obtaining consent from the patients. Pre-operative evaluation data forms were completed and all relevant preoperative scores were recorded. Postoperative data was collected during follow up and through postal questionnaires. The timescale was at 6 months and 1 year. Evaluation of outcome measures included Oxford shoulder score (OSS), Quick DASH (QD) and European Quality of Life 5Dimensions 3L (EQ 5 D).

\section{Inclusion and exclusion criteria}

All patients with clinical and radiological diagnosis of SIS, who had a failed conservative treatment and underwent ASAD, at Ninewells Hospital, Dundee, UK, and Perth Royal Infirmary, Perth, UK, and Stracathro Hospital, UK during the period April 2015 to January 2017. Patient who had given their consent to be part of the study were included. Exclusion criteria were patient with full thickness tears of the rotator cuff, internal rotation contractures, previous open shoulder surgeries, gleno-humeral instability, inadequate follow-up, biceps tendinitis or tear, deltoid weakness, neurological involvement and patients unwilling to consent to be part of the evaluation programme.

\section{Statistical Analysis}

Statistical Package for Social Sciences (SPSS) for windows (version 22) was used for all statistical analysis in this study for processing of the data. Repeated measure analysis of variance (ANOVA) was used to analyse patient related outcome measures (PROMs) function, the total score preoperatively and post-operatively at 6 months and at one year follow up for EQ 5 D, OSS and Quick DASH. Chi Square test was used for comparison of postoperative score between different groups of patients. The statistical significance level was set at value of $p<0.05$. Post hoc test was used to evaluate the statistical significance of the means.

\section{Results}

The mean age of patients included in the study was 56.52 years with a range from 32 years to 81 years. There were 27 females and 23 males included in the study, $60 \%$ patients had right sided pathology and $40 \%$ had a left sided pathology. The BMI of the patients included in the study was within a range of 18 to 41 with a mean value of 28 . Patients were divided into 3 groups based on their BMI. Patients with BMI less than 25 were in group 1 , with 25 to 30 were in group 2 and those with more than 30 were in group 3 . Impingement was more commonly diagnosed in male patients. Whereas, the diagnosis of impingement and acromioclavicular arthritis were most common in female patients.

\section{European Quality of Life 5 Dimensions (EQ 5 D)}

EQ 5 D score was recorded preoperatively, at 6 months and 1 year post-operatively. EQ VAS and EQ TTO components of the score were analysed. Mean EQ VAS and EQ TTO score in the study group improved from preoperatively to postoperatively at 6 months and 1 year as shown in figure 1 . There was statistically significant difference of $\mathrm{p}=0.000$ between preoperative score and 6 months postoperative scores. Similarly, there was statistically significant difference of $\mathrm{p}=0.000$ between preoperative score and 1 year postoperative scores. There was no significant difference, $\mathrm{p}=0.744$, observed between 6 months and 1year postoperatively.

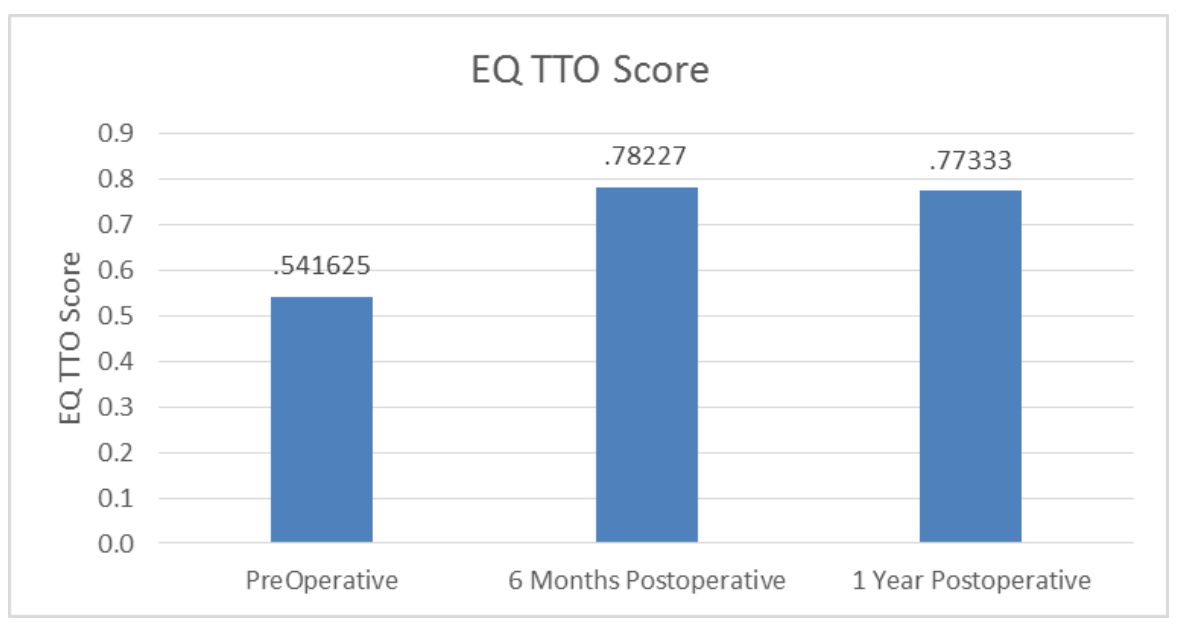

Fig 1: Mean EQ TTO Scores preoperative and postoperative 
Oxford shoulder score

Mean OSS score in the study group was 27.894 preoperatively, which improved to 39.988 at 6 months postoperatively and 40.348 at 1 year postoperatively as shown in figure 2.

There was statistically significant difference of $\mathrm{p}=0.000$ between preoperative score and 6 months postoperative scores. Similarly, there was statistically significant difference of $\mathrm{p}=0.000$ between preoperative and 1 year postoperative scores. There was no significant difference, $\mathrm{p}=0.651$ noted between 6 months and 1year postoperatively.

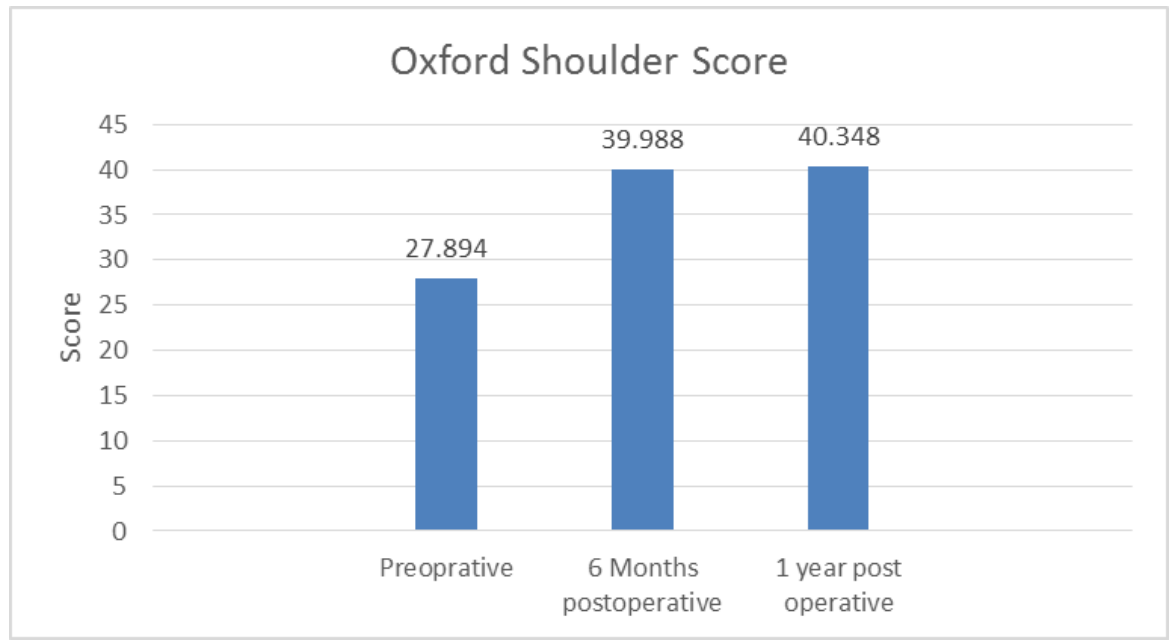

Fig 2: Mean OSS preoperative and postoperative

\section{Quick DASH Disability score}

Mean QD Disability score in the study group was 47.496preoperatively, which improved to 20.241at 6 months postoperatively and 20.241 at 1 year postoperatively as shown in figure 3. There was statistically significant difference of $\mathrm{p}=0.000$ between preoperative scores and 6 months postoperative scores. Similarly, there was statistically significant difference of $\mathrm{p}=0.000$ between preoperative and 1 year postoperative scores. No significant difference $(p=0.996)$ was noted between 6 months and 1year postoperatively.

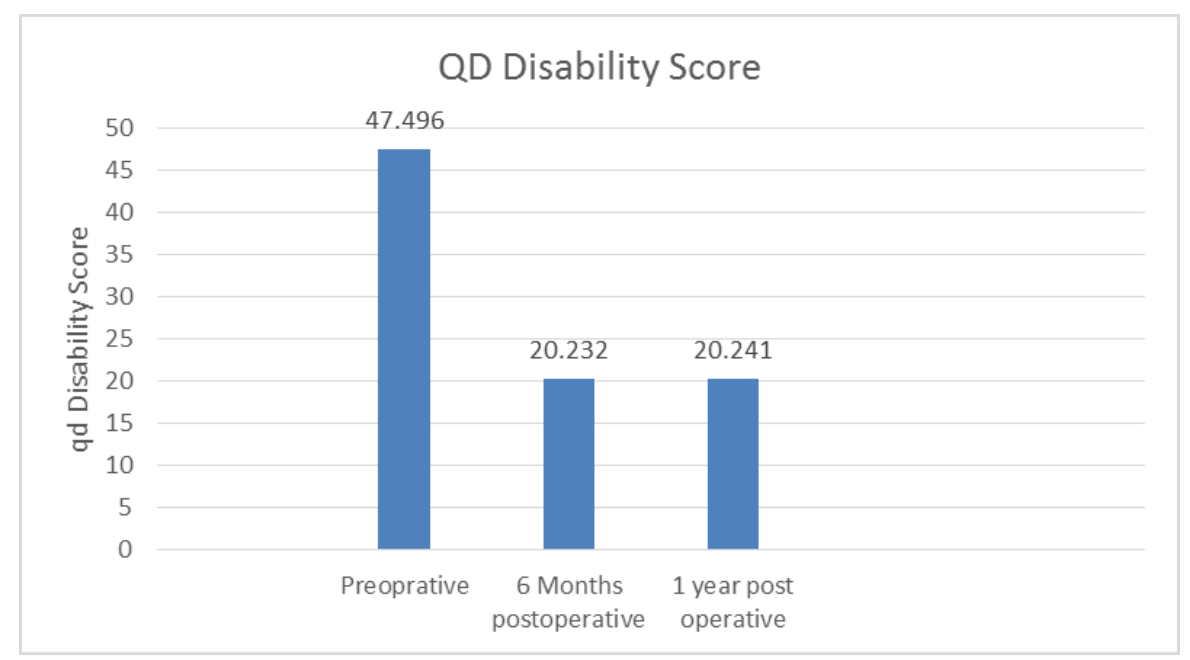

Fig 3: Mean QD Disability score preoperative and postoperative

Table 1: Mean PROMs scores preoperative and postoperative

\begin{tabular}{|c|c|c|c|}
\hline Scores & Preoperative & $\begin{array}{c}6 \text { Months } \\
\text { Postoperative }\end{array}$ & $\begin{array}{c}1 \text { Year } \\
\text { Postoperative }\end{array}$ \\
\hline $\begin{array}{c}\text { Mean EQ } \\
\text { VAS Score }\end{array}$ & 69.388 & 75.776 & 77.102 \\
\hline $\begin{array}{c}\text { Mean EQ } \\
\text { TTO Score }\end{array}$ & 0.54162 & 0.78227 & 0.77333 \\
\hline $\begin{array}{c}\text { Mean } \\
\text { OSS sore }\end{array}$ & 27.894 & 39.988 & 40.348 \\
\hline $\begin{array}{c}\text { Mean QD } \\
\text { Disability } \\
\text { score }\end{array}$ & 47.496 & 20.232 & 20.241 \\
\hline $\begin{array}{c}\text { Mean QD } \\
\text { Work score }\end{array}$ & 48.125 & 22.083 & 21.25 \\
\hline $\begin{array}{c}\text { Mean QD } \\
\text { Sport score }\end{array}$ & 78.309 & 44.485 & 38.603 \\
\hline
\end{tabular}

\section{Demography}

The study group was divided into two groups one with age less than 50 years and the other with age 50 years and above. Assessment of PROMs scores in these groups showed better improvement in mean EQ VAS, EQ TTO, OSS and Quick DASH score in patients with age less than 50 year. The difference was not significant $\mathrm{p}=0.097$ in patients aged 50 year or more. Females had shown better average postoperative mean PROMs scores compared to males.

Patients with BMI less than 25 had better improvement of EQ TTO scores postoperatively at 6 months and 1 year when compared with patients with higher BMI. No significant difference in EQ VAS score was observed between preoperative and postoperative scores at 6months and 1 year in patients with BMI of 25 or more. Patients who underwent 
left shoulder ASAD demonstrated higher improvement in mean scores as compared to their counterparts who were operated on the right shoulder.

An analysis of the PROMs scores in patients with comorbidities in the study group was done. $73 \%$ of patients who had comorbidities, suffered from diabetes and hypertension, $18 \%$ were smokers and $9 \%$ had a previous history of trauma. Reduction in mean scores was observed when compared to patients without comorbidities. Out of 50 patients, 22 where working, 11 of these were manual workers the remainders were office workers. Average mean scores were better in patients who were not working than those working. Manual workers had poor improvement in this study group.
Patient satisfied and dissatisfied at 6 months postoperatively

PROMs scores were assessed in patients who reported satisfaction or dissatisfaction from ASAD surgery at 6 months postoperatively. $72 \%$ of patients reported to be satisfied at 6 months postoperative after ASAD surgery, 26\% reported to be dissatisfied and $2 \%$ did not comment on their satisfaction as seen in figure 4.Significant reduction in the mean PROMs scores was noted in dissatisfied patients at 6 months and 1 year postoperatively. Statistically significant difference, $\mathrm{p}=0.048$, between PROMs scores preoperatively and 6 months postoperatively was observed in dissatisfied patients.

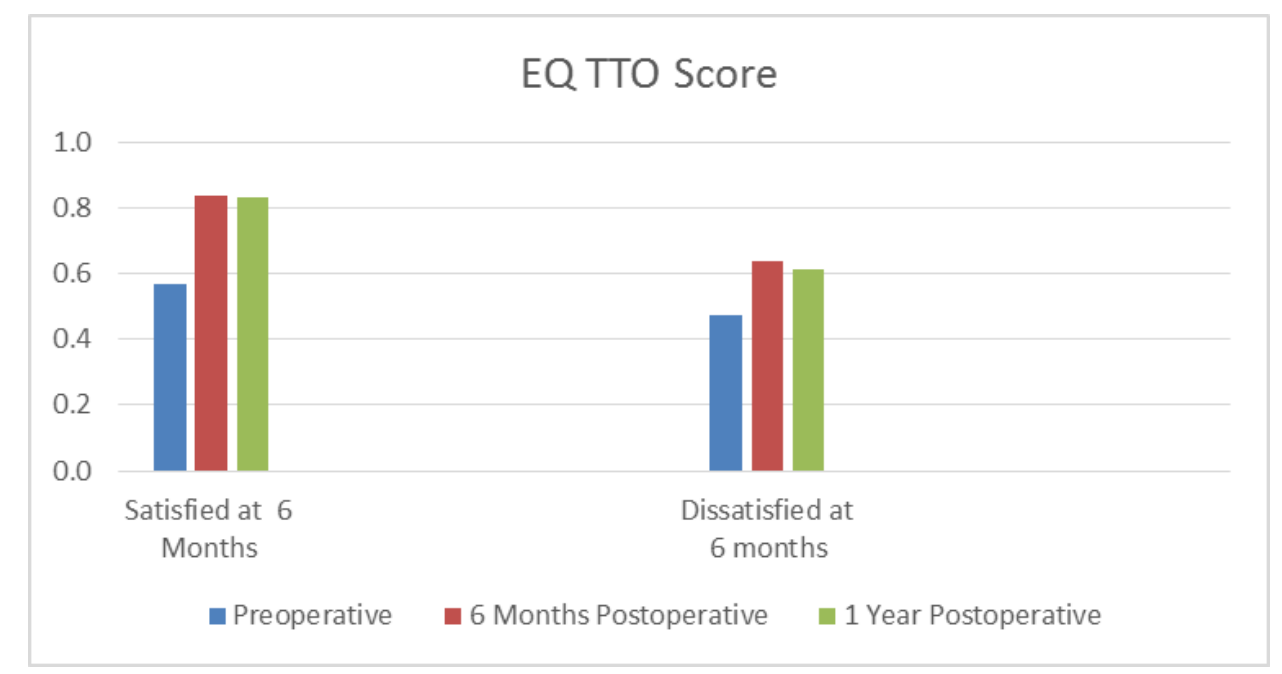

Fig 4: Mean EQ TTO score in satisfied patients at 6 months postoperative.

Patient satisfaction and dissatisfaction at 1 Year postoperative

PROMs scores were assessed in patients who reported satisfaction or dissatisfaction from ASAD surgery at 1year postoperatively. $68 \%$ of patients reported to be satisfied at 1 year postoperative after ASAD surgery, 30\% reported to be dissatisfied at 1 year after ASAD surgery and 2\% did not comment on their satisfaction as seen in figure 5 . There was an increase of $4 \%$ in dissatisfied patient at 1 year in comparison to 6 month postoperative satisfaction group.

Significant reduction in the Mean PROMs scores was noted in dissatisfied patients at 6 months and 1 year postoperatively. Statistically significant difference between PROMs score preoperatively and 6 months postoperatively was observed in patients who were dissatisfied at 6 months after surgery.

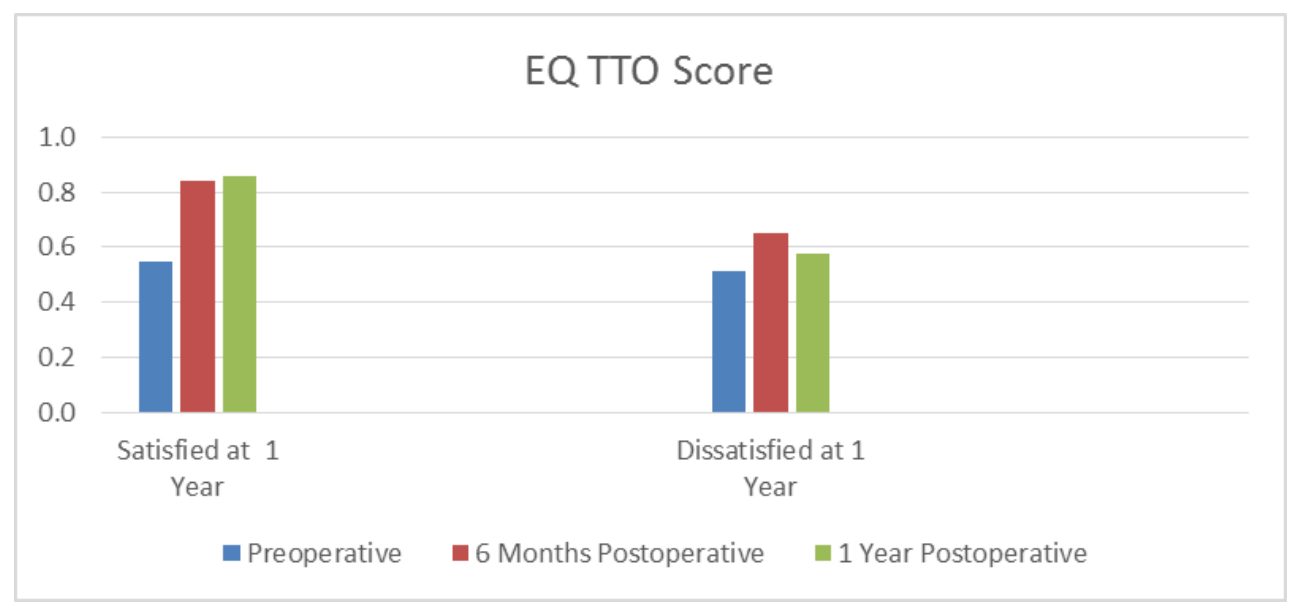

Fig 6: Mean EQ TTO score indifferent patient satisfaction groups at 1 year.

\section{Discussion}

Literature shows that there was considerable increase in the number of subacromial decompression being performed in NHS, England. ${ }^{[8]}$ However, recent studies have raised doubts over the effectiveness of ASAD compared with a supervised structured exercise programme over a period of time. ${ }^{[4]}$ In patients who fail conservative treatment and physiotherapy programme, surgery was frequently the only recourse. ASAD has become a widely used technique for the treatment of SIS and has been shown to be efficient, durable and provides a 
high patient satisfaction. [9]

PROMs are recognised as the way forward in outcome studies as they include involvement of the patient's perception of their outcome. The current study analysed the outcome of ASAD based on the patient perception of their outcome and therefore eliminate the clinician's assessment bias. All 50 cases included in the study had $100 \%$ return rate of data at 6 month period and 1 year postoperative period.

European Quality of Life 5 Dimensions 3L (EQ 5D) score It was noted that the average mean scores in all the patients improved from the preoperative period to 6 months postoperatively. The mean EQ 5D 3L score continued to improve up to 1 year postoperatively in these patients.

The VAS score showed better improvement than the TTO score. This suggested that pain and quality of life improved after the procedure up to 6 month postoperatively and continued up to 1 year postoperatively. There was however, no statistically significant difference noted in scores between 6 months postoperative and 1 year postoperative period in our study $(p=0.744)$. This suggested that the maximum improvement in the EQ 5D 3L scores were achieved at 6 months postoperatively. However, there was negligible improvement in EQ VAS scores and a marginal decrease in EQ TTO scores from 6 month postoperative to 1 year postoperative period

\section{Oxford shoulder score}

Oxford shoulder score in the present study showed significant improvement in the Mean postoperative scores at 6 months and 1 year postoperative when compared with Mean preoperative scores $(p=0.000)$. However, no statistically significant difference was noted between 6 months and 1 year postoperative values $(\mathrm{p}=0.644)$. This suggested that the greatest improvement in the functional outcome was in the initial 6 months and a modest gain at 1 year postoperatively. This was comparable to the studies by Singh et al. ${ }^{[10]}$ and Magaji et al. ${ }^{[8]}$

\section{Quick DASH score}

The current study showed statistically significant improvement in the mean Quick DASH score between preoperative and postoperative values $(p=0.00)$. No statistically significant difference was noted between 6 month and 1 year postoperatively $(p=0.996)$. This suggested that patient pain and function improved at 6 month postoperative but not at 1 year. This was similar to the results of Allom et al ${ }^{[6]}$ and Bengtsson et al. ${ }^{[11]}$ This suggested that the maximum improvement in the PROMs scores were achieved at 6 months postoperatively. However, there was negligible improvement from 6 month postoperative to 1 year postoperative period.

\section{Patient satisfaction at 6 month postoperative and 1 year postoperative}

Patient satisfaction was assessed at 6 months and 1 year postoperatively, patients who had reported to be satisfied with surgery were compared to patients who were dissatisfied. The assessment of PROMs scores at 6 months and 1 year postoperatively showed that the group of patients who were dissatisfied with outcome at 6 months and 1 year postoperative scored far less than the group of patients who were satisfied with the outcome. Thus, it was observed that in spite of marginal improvement in mean postoperative PROMs score as compared to preoperative PROMs score, patients still reported to be dissatisfied from the outcome of surgery.
An increase in the total number of dissatisfied patients by $4 \%$ was noted at 1 year postoperative. Only 3 patient out of the 12 dissatisfied patient at 6 months became satisfied at 1 year postoperative. Whereas, 5 patients who were satisfied at 6 months were reported to be dissatisfied at 1 year postoperative. This suggested that the patients who were dissatisfied at 6 month after surgery did not improve much at 1 year postoperative period.

The reason for dissatisfaction as reported by these patients were pain, stiffness, limited mobility, slow progress, ongoing symptoms and not matching the expected outcome of the surgery. Pain being the most common reason for dissatisfaction. This was similar to the study by Bengtsson et al, ${ }^{[11]}$ Pillai et al. ${ }^{[12]}$ Razmjou et al ${ }^{[13]}$ stated that satisfaction was a complex concept related to multiple factors, majority of patients in their study had unrealistic expectations. The level of satisfaction reported was not directly affected by the existence of identifiable cause of failure.

\section{Complications}

Complications were recorded in 4 patients postoperatively, 2 of who had developed frozen shoulder within 6 months postoperatively and the other 2 had developed rotator cuff tear within in 1 year following surgery. No significant difference was noted in preoperative and postoperative PROMs scores in these patients. Ongoing pain was seen in 2 patients, one received a steroid infiltration at 6 months and the other had a physiotherapy referral.

\section{Conclusion}

This study demonstrated significant improvement in mean postoperative PROMs scores at 6 months and 1 year postoperative which was statistically significant. Maximum improvement in PROMs was observed at 6 months postoperatively. Decreased improvement in mean PROMs was observed from 6 months to 1 year postoperatively Improvement in PROMs postoperatively was better appreciated in female gender, patients with low BMI, left shoulder pathology and in patients aged less than 50 years. It was observed that patients who do not show satisfactory improvement in PROMs scores at 6 months postoperatively had continued to score less at 1 year postoperative. Pain was reported to be the most common reason for dissatisfaction in this study group.

PROMs are recognised as the way forward in outcome studies as they include involvement of the patient's perception of their outcome. The current study analysed the outcome of ASAD based on the patient perception of their outcome and therefore eliminate the clinician's assessment bias. This study suggested that ASAD for impingement gives a high patient satisfaction after surgery with significant improvement in function and quality of life.

\section{References}

1. Patel V, Singh D, Calvert P, Bayley J. Arthroscopic subacromial decompression: Results and factors affecting outcome. Journal of Shoulder and Elbow Surgery. 1999; 8(3):231-237.

2. Urwin M, Symmons D, Allison T, Brammah T, Busby H, Roxby $\mathrm{M}$ et al. Estimating the burden of musculoskeletal disorders in the community: the comparative prevalence of symptoms at different anatomical sites, and the relation to social deprivation. Annals of the Rheumatic Diseases. 1998; 57(11):649-655.

3. Judge A, Murphy R, Maxwell R, Arden N, Carr A. 
Temporal trends and geographical variation in the use of subacromial decompression and rotator cuff repair of the shoulder in England. The Bone \& Joint Journal. 2014; 96-B(1):70-74.

4. Butt U, Whiteman A, Wilson J, Paul E, Roy B. Does arthroscopic subacromial decompression improve quality of life. The annals of the royal college of surgeons of England. 2015; 97(3):221-223.

5. Howie CR, Hamilton DF. PROMs- How was it for you ? Journal of Trauma and Orthopaedics, 2013; 1(1):49-51.

6. Allom R, Colegate-Stone T, Gee M, Ismail M, Sinha J. Outcome analysis of surgery for disorders of the rotator cuff: A comparison of subjective and objective scoring tools. Journal of Bone and Joint Surgery - British Volume. 2009; 91-B(3):367-373.

7. Hultenheim Klintberg I, Karlsson J, Svantesson U. Health-related quality of life, patient satisfaction, and physical activity 8-11 years after arthroscopic subacromial decompression. Journal of Shoulder and Elbow Surgery. 2011; 20(4):598-608.

8. Magaji S, Singh H, Pandey R. Arthroscopic subacromial decompression is effective in selected patients with shoulder impingement syndrome. The Bone \& Joint Journal. 2012; 94-B(8):1086-1089.

9. Luyckx L, Luyckx T, Donceel P, Debeer P. Return to work after arthroscopic subacromial decompression. ActaOrthop Belg. 2011; 77(6):737-42.

10. Singh H, Mehta S, Pandey R. A preoperative scoring system to select patients for arthroscopic subacromial decompression. Journal of Shoulder and Elbow Surgery. 2014; 23(9):1251-1256.

11. Bengtsson M, Lunsjö K, Hermodsson Y, Nordqvist A, Abu-Zidan F. High patient satisfaction after arthroscopic subacromial decompression for shoulder impingement: A prospective study of 50 patients. ActaOrthopaedica. 2006; 77(1):138-142.

12. Pillai A, Eranki V, Malal J, Nimon G. Outcomes of Open Subacromial Decompression after Failed Arthroscopic Acromioplasty. ISRN Surgery. 2012; 2012:1-5.

13. Razmjou H, Lincoln S, Axelrod T, Holtby R. Factors Contributing to Failure of Rotator Cuff Surgery in Persons with Work-Related Injuries. Physiotherapy Canada. 2008; 60(2):125-133. 